\title{
INVESTIGATION OF SOLID-LIQUID PHASE TRANSITION FOR Sn-54wt\%In ALLOY BY POSITRON ANIHILATION SPECTROSCOPY
}

\author{
St. ChabiK ${ }^{a}$, M. Szuszkiewicz ${ }^{a, b}$ AND W. RudzińsKa ${ }^{a}$ \\ ${ }^{a}$ Institute of Physics, University of Opole, Oleska 48, 45-052 Opole, Poland \\ ${ }^{b}$ Institute of Experimental Physics, University of Wrocław \\ M. Borna 9, 50-204 Wrocław, Poland
}

The peak coincidence counting rate $F(T)$ was measured for Sn-54wt\%In alloy at the temperature range from room temperature to $400^{\circ} \mathrm{C}$. It was observed that for both heating and cooling run, $F(T)$ parameter changes in the liquid phase. These changes were related to disintegration of SnII microcrystals. From a simple trapping model the defect disintegration enthalpy, $I_{1}$, was calculated. For heating and cooling run, the value of $H_{1}$ was $0.53 \pm 0.03 \mathrm{eV}$ and $0.67 \pm 0.07 \mathrm{eV}$, respectively. For well-annealed and cold rolled samples we find only one component, equal $200 \mathrm{ps}$, in the lifetime spectrum.

PACS numbers: 64.70.Dv, 61.72.Cc, 78.70.Bj.

\section{Introduction}

Positron annihilation spectroscopy (PAS) is very successful in investigation of defects in the solid state samples. PAS has rather rarely been used for studies of solid-liquid phase transition. There are two reasons for this: the first - experimental difficulties, the second - lack of a theoretical description of the behaviour of positrons in liquid metals. The vacancy model of melting [1] and the microcrystalline liquid model [2] predict that the structure of metals at the melting point, $T_{m}$, is strongly defected. Thus saturation of positron annihilation should be observed. Many experimental data seem to confirm this suggestion. For example for $\mathrm{Al}, \mathrm{Pb}[3]$ and $\mathrm{Ni}[4]$ an increase about 3 percent in peak coincidence counting rate $F(T)$, has been observed at the melting point. In the liquid phase the value of $F(T)$ parameter has been constant. Similar changes of $F(T)$ have been observed for indium [3].

However, for such metals as $\mathrm{Ga}$ and $\mathrm{Sn}$ annihilation parameters dramatically change at the melting point. For Sn the mean positron diffusion length [5], $F(T)[6]$ and the doppler broadening $S$ parameters [7] jump at the melting point. Similar 
results have been obtained for $\mathrm{Ga}[5,8]$, where the values of the positron lifetime in the solid and liquid state are different, too [9]. This behaviour is characteristic of the metal with a very complex melting process. Some experimental data from other than PAS methods have indicated that for tin at $T_{m}$ two solid phases (SnI and SnII) coexist in equilibrium with the melt [10-12]. SnII has been identified as a body centred tetragonal, but with much lower $c / a$ ratio than SnI [13]. At the atmospheric pressure two liquid species exist in the molten in the temperature range between $T_{m}\left(232^{\circ} \mathrm{C}\right.$ ), and about $420^{\circ} \mathrm{C}$, at which SnII vanishes [14] (second melting of tin). For indium at $T_{m}$, microcrystals in short range ordering exist as well. Their contribution has been estimated as equal to 31 percent [15], but a crystal structure of microcrystals insignificantly differs from that of the solid state [16]. Consequently, the annihilation parameters for tin dramatically rise at $T_{m}[5-7]$, whereas for indium positron lifetime [17] and mean positron diffusion length [5] do not change.

$\mathrm{Sn}-54 \mathrm{wt} \%$ In alloy is nearly an eutectic composition. During the solidification process two separate tin and indium crystals are growing. It is interesting to find out how individual characteristics of tin and indium influence the annihilation process.

\section{Experiment}

The sample was prepared by melting the granulates of the $4 \mathrm{~N}$ pure tin and indium in the rectangular measuring vessel. The heater was placed on the one side of the vessel, while on the other side the rectangular titanium foil ( $8 \mu \mathrm{m}$ thick) was mounted. This way positrons from ${ }^{22} \mathrm{Na}$ source penetrated the interior of the sample through the window. After melting the sample was stored at $400^{\circ} \mathrm{C}$. Next, during slow cooling (heating) step by step, the peak coincidence counting rate was measured. At each temperature the number of coincidences accumulated in few hours was of about $5 \times 10^{4}$. The measurements were performed in the protective argon atmosphere under atmospheric pressure. Details of the apparatus are given in Ref. [8]. A positron lifetime spectrum was measured at room temperature for the well-annealed and the cold rolled sample. A conventional fast-slow spectrometer was used with a resolution function (FWHM) equal 250 ps.

\section{Results and discussion}

The results of the measurements are shown separately for the heating (Fig. 1a) and cooling (Fig. 1b) run, because there is a small probability that the conditions of thermal equilibrium at the given temperature are the same when the temperature is increasing or decreasing. Solidus and liquidus temperatures, $T_{s}, T_{l}$, respectively, are slightly different and are marked on Figs. $1 \mathrm{a}$ and $1 \mathrm{~b}$.

As it is shown the saturation of annihilation in the solid and liquid phase (slightly above $T_{l}$ ) occurs and then the value of $F(T)$ parameter decreases. Thus, experimental data have been elaborated on the basis of the following assumptions:

(i) In the liquid phase, near $T_{l}$, positrons are trapped at defects (saturation of annihilation); 

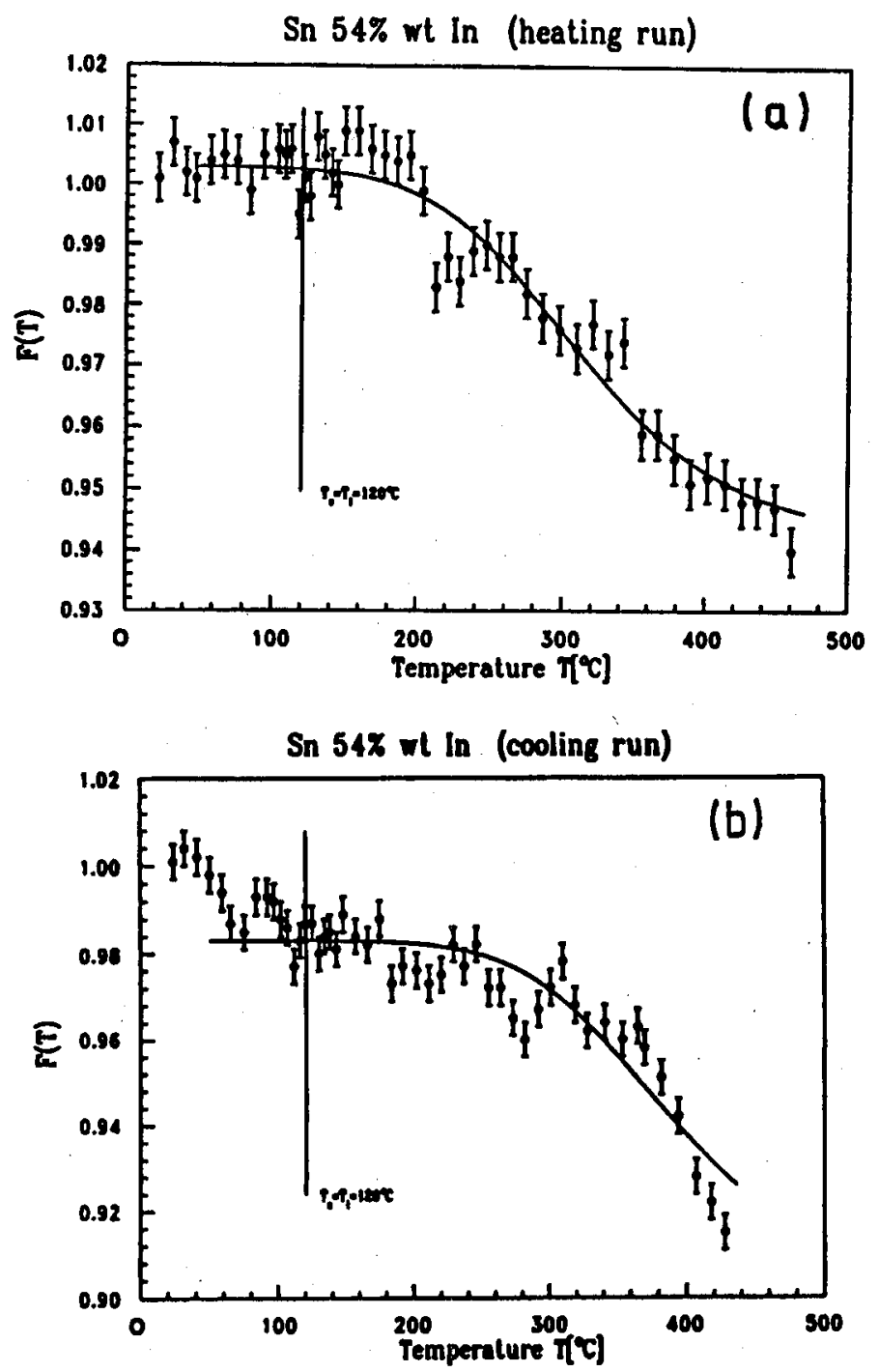

Fig. 1. (a) Dependence of peak coincidence counting rate vs. temperature of Sn-54wt\%In alloy for heating run, (b) the same as in Fig. 1a, but for cooling run.

(ii) Together with the rise of temperature the size of microcrystals of SnII decreases;

(iii) Above $400^{\circ} \mathrm{C}$ positrons annihilate in the disordered liquid tin. Thus, the temperature dependence of $F(T)$ in the liquid phase can be described with the use of the "inversed" trapping model 


$$
F(T)=\frac{F_{l}+F_{1} A_{1} \exp \left(H_{1} / k T\right)}{1+A_{1} \exp \left(H_{1} / k T\right)}
$$

where $F_{l}, F_{1}$ - average values of $F(T)$ near $T_{l}$ and $400^{\circ} \mathrm{C}$, respectively, $k-$ Boltzmann's constant, $H_{1}$ - defect disintegration enthalpy. The constant $A_{1}=$ $c_{l} \kappa_{l} / \tau_{d}$ characterizes detrapping of positrons from defects. $c_{l}$ is the concentration of defects at $T_{l} ; \kappa_{l}$ denotes the specific positron detrapping rate per unit defect concentration; $\tau_{d}$ - positron lifetime in defects. Additionally, the assumption has been made that the concentration of defects changes according to the formula $c(T)=c_{l}\left[1-\exp \left(H_{1} / k T\right)\right]$ and $\exp \left(H_{1} / k T\right) \gg 1$ at $T_{l}$.

The best fitted curves (solid lines in Figs. $1 \mathrm{a}$ and $1 \mathrm{~b}$ have been obtained for $H_{1}=0.53 \pm 0.03 \mathrm{eV}$ and $A_{1}=2.5 \times 10^{-5}$ when temperature increases, and $H_{1}=0.67 \pm 0.09 \mathrm{eV}, A_{1}=7.5 \times 10^{-5}$ (temperature decreases), respectively. From our measurements it is not clear what the nature of the positron trapping centres is. However, it may be assumed that they are connected with microcrystals of tin, because a similar dependence as for the alloy has been observed in the case of liquid tin [6], but not for SnIn alloys containing less than $50 \%$ of In [18]. Also, it is worth to notice that the value of $H_{1}$ is almost the same as the monovacancy formiation enthalpy for pure tin. Thus, it may be concluded that positrons are trapped at vacancy-like defects of SnII. On the other hand, unexpected saturation of annihilation between solidus temperature and room temperature is evident in Figs. $1 \mathrm{a}$ and $1 \mathrm{~b}$. At this temperature range the positrons are trapped probably at the boundary of the grains. It is quite impossible that the SnII crystals are present in the solid Sn-54wt\%In alloy, because as it has been shown in Ref. [13], for pure tin, at room temperature, SnI transforms to SnII at $110 \mathrm{kbar}$ pressure. From the positron lifetime spectrum at room temperature, after subtraction of the contribution of the positron source and the kapton foil, one component has been obtained with $\tau=200.7 \pm 0.4 \mathrm{ps}$. After cold rolling (50\% reduction of thickness) the positron lifetime spectrum of Sn-54wt\%In does not change.

\section{Conclusions}

The investigation of many properties of liquid metals is rather difficult. Only a small number of methods, applicable to the studies of defects in solid phase, may be used in case of liquid metals. As we have shown, PAS may be used to determine the defect parameters in the liquid $\mathrm{Sn}-54 \mathrm{wt}$ \% In alloy. We do not know examples of the application of PAS and the trapping model to the studies of defects in liquid metals. Nevertheless, the values of $H_{1}$ and $A_{1}$ calculated by us are reasonable. It is worth noting that high accuracy of measurement is necessary, because the value of $F(T)$ parameter changes only about $3 \%$ for liquid Sn-54wt\%In alloy. This is different in comparison to simple solid metals, where annihilation parameters change up to ten percent between the threshold temperature and the melting point. Finall: we do not know to what extent lie specific properties of the eutectic alloy influence the results of our measurements. Therefore, investigations of another composition of SnIn alloy with higher content of In are necessary. 


\section{References}

[1] T. Górecki, High Temp.-High Press. 15, 115 (1983).

[2] W.I. Arharov, I.A. Novohatski, Dokl. Akad. Nauk USSR 185, 1069 (1969).

[3] W. Triftshauser, Phys. Rev. B 12, 4634 (1975).

[4] M.J. Fluss, L.C. Smedskjaer, B. Chakraborty, M.K. Chason, J. Phys. F, Met. Phys. 13, 817 (1983).

[5] K. Lynn, in: Proc. "Enrico Fermi" Intern. School of Physics on Positron Spectroscopy of Solids, Varenna 1993, to be published.

[6] W. Rudzińska, Cz. Szymański, St. Chabik, B. Rozenfeld, in: Proc. European Meeting on Positron Studies of Defects (PSD-87), Eds. G. Dlubek, O. Brümmer, G. Brauer, K. Henning, Martin-Luther-Universität, Halle-Wittenberg 1987, Vol. 2, Part 1, p. B6.

[7] N. Shah, A.L. Catz, Phys. Rev. B 30, 2498 (1984).

[8] Cz. Szymański, St. Chabik, J. Pająk, B. Rozenfeld, Phys. Status Solidi A 60, 375 (1980).

[9] W. Brandt, H.F. Wuang, Phys. Lett. A 27, 700 (1968).

[10] A.W. Ewald, E.E. Kohake, Phys. Rev. 97, 607 (1955).

[11] A.M. Alper, Phase Diagrams, Vol. 1, Academic Press, New York, London 1970, p. 265.

[12] G.C. Vezzoli, Mater. Res. Bull. 23, 1 (1988).

[13] J.C. Jamieson, in: Production and Measurement of High Temperature, Ed. J.L. Margrave, Gordon and Breach, New York 1964, p. 101.

[14] G.S. Vezzoli, High Temp.-High Press. 20, 679 (1988).

[15] W.L. Ladianov, W.J. Arharov, I.A. Novohatski, W.Z. Kisunko, Fiz. Met. Metalloved. 34, 10 (1972).

[16] A.W. Romanova, B.A. Melnik, Ukr. Fiz. Zh. 15, 101 (1970).

[17] W. Eckert, I. Bringman, H.E. Schaefer, Condens. Matter 1, Suppl. A (1989).

[18] St. Chabik, W. Rudzińska, M. Szuszkiewicz, Cz. Szymański, Acta Phys. Pol. A 83, 261 (1993). 